\title{
Ethical Issues for Older People in the Emergency Department
}

\section{Introduction}

Despite variations in the configuration of healthcare services across Europe, the Emergency Department is often seen as 'The Front Door' to secondary care services at times of crisis. By their nature, Emergency Departments are designed to triage and rapidly assess attendees, initiating treatment, referring to specialist services or facilitating discharge. Although this model is highly effective for those with a single-organ problem, the complex and often subtle presentations characteristic of frail older people, demand a more holistic approach.

The risk-benefit ratio associated with decision making in frail older people in the ED is not the same as for more 'robust' cohorts. For example, whilst hospital can be viewed as a place of safety, enforced bed-rest can lead to reduced muscle mass and increase the risk of adverse outcomes such as falls(1). In people aged $85+$, admission to hospital is associated (but not causally) with a mortality rate of $46 \%$ at one year(2); some of this might be avoidable. Factors influencing such outcomes in frail older people include the existence of cognitive impairment, multiple co-morbidities, polypharmacy and concomitant functional impairment, which makes assessment and management challenging.

These issues conflate to influence decision making with frail older people in the ED setting. An ethical framework can be helpful in guiding the decision making process. In this review we will discuss particular challenges for older people in the ED and the ethical implications.

\section{The ethical principles governing decision making}

One of the most commonly used frameworks in medical ethics is Beauchamp \& Childress' Four Principles (3). These consist of autonomy (the right of an individual to make decisions), beneficence (acting in the patient's best interests), non-maleficence (acting to do no harm), and justice (fairness and equity). Whilst ethical principles are designed to be used in any setting, this article develops their application in clinical practice in the Emergency Department. When considering ethical dilemmas it can be helpful to consider each of these principles in turn.

Case Study: Mrs A, an 86 year old lady, is bought into the Emergency Department with suspected sepsis. Staff are concerned that she is confused and may be delirious, and she is refusing to have an intravenous (IV) cannula inserted for IV antibiotics and fluids. You have been asked to cannulate Mrs A. How would you approach this scenario?

Autonomy: Wherever possible, an individual should be helped and encouraged to make their own decision and these decisions should be respected. In this case however, Mrs A may not have capacity to make this decision as she is potentially delirious. The principle of autonomy is strongly linked to capacity, which is discussed in detail later.

Beneficence: Healthcare professionals should always try to act with the patient's best interests in mind. In most European countries, if a patient has capacity then their decision cannot be overridden, even if it is felt that their decision is unwise. If the patient does not have capacity for the specific issue in question, then healthcare professionals should initiate a discussion with those that know the patient well, as well as make reference to any pre-existing statement of preference or values (e.g. Advance Directive) to describe what the patient would have wanted (best interests). In this scenario, if Mrs A has capacity then her decision not to have the IV cannula inserted would have to be respected. If she does not have capacity, then a decision needs to be taken in her best interests. This would need to take account of the procedure itself 
(having a cannula inserted can be painful and may be traumatic in the short term if Mrs A resists the procedure), as well as considering her prior expressed views as regards treatments. It might be that Mrs A has a long-established preference for avoiding hospital treatment, and has stated that she would prefer to die than be subject to acute hospital care. On the other hand, she might have been planning to attend a daughter's wedding or other such important event, and might want all possible treatment to allow that to happen. These factors need to be explored and understood in order to arrive at a best interests decision.

Non-maleficence: Perhaps the most obvious of the four ethical principles is that as healthcare professionals we should do no harm to patients. Inserting an IV cannula carries certain risks such as infection or damage to the skin. However, not treating suspected sepsis with IV antibiotics and fluids would potentially cause much greater harm and could lead to long-term disability or even death. It is important to use a physician's body of knowledge to provide a 'risk-benefit analysis' of the pros vs. cons of an intervention.

Justice: The principle of justice, that we should act fairly and equitably, means that all individuals should have equal opportunities to receive care appropriate for their condition. Older people with frailty often exhibit differential challenge - namely that most in need are least able to access services. This might be due to sensory deprivation impairing communication, or disability leading to reduced access to care. Or it may be that services are not appropriately configured and attuned to the needs of older people with frailty. An ethical service will be cogniscent of these issues and make provision for the more vulnerable members of society accordingly.

\section{Assessing capacity}

\section{Definition of capacity}

In English law, capacity is defined by the Mental Capacity Act (MCA) 2005(4) as a decision specific ability to understand, weigh and retain information and verbalise one's ideas and preferences. The MCA proposed that individuals lacking mental capacity should be enabled to exercise their extant (or remaining) decision-making capacity(5). This legal definition does not give specific measures of an individual's ability to comprehend information. Rather, it is acknowledges that capacity is dynamic and that the 'threshold' varies according to the gravity of the decision to be taken. Transient circumstances such as delirium, tiredness, dysphasia etc. can hinder an individual's thinking process and ability to make a sound judgement or engage in a task. Equally, the capacity threshold for apparently simple issues such as a choice of clothing or food, will not be the same as decision about life-sustaining treatment, even in the same individual. Therefore capacity should be examined in the context of the specific issue at hand. In the ED context, acute conditions such as pain can affect capacity and competence directly. The MCA acknowledges the possibility of fluctuations in capacity, and reminds decision makers that reassessment may be necessary at a later stage - or deferment of non-urgent decision until capacity can be reassessed.

\section{Capacity assessment}

Issues surrounding capacity and consent may arise in any age of patient in any healthcare setting and of course do not just apply to older people in the emergency department. However, these issues are particularly relevant in the older population as they are more likely to suffer from conditions which may impair capacity, such as delirium or stroke. They are also more likely to have conditions which make it more challenging to assess capacity such as hearing or sight impairment and communication difficulties. Addressing such matters in emergency departments can also be more difficult than in other healthcare settings because decisions are being made by professionals who have never met the patient before, in an environment which is often noisy and time-pressured. 
Assessing capacity properly and appropriately is crucial because deciding that somebody lacks capacity and enacting the best interest's framework impacts upon autonomy (see below on best interests). All health and social care professionals should be trained to assess patients' capacity for the treatments that they might offer; high stakes decision might require the involvement of specialists in capacity assessment, such as psychiatrists.

Capacity is a time-specific and decision-specific assessment. It must never be assumed that because a patient has a potentially impairing condition such as dementia, that they do not have the capacity to make a decision. Every effort should be made to help the patient to give informed consent. This may include providing written information, using sign language, finding the patient's glasses and hearing aids and taking the time to understand, and be understood. In the case of delirium, characterised by fluctuations, the need to re-evaluate capacity is key. This is particularly relevant if healthcare professionals feel there is a need to use restrictive measures to keep the individual safe, e.g. preventing them from leaving the department. Such actions, although generally well intentioned, must be balanced against the individual's rights and freedoms. Healthcare professionals have a responsibility to familiarise themselves with the relevant legislation and are encouraged to seek advice in difficult situations.

Assessing patients with confusion

It may also be more difficult to thoroughly assess patients who are confused as they may not be able to give a comprehensive history of events, symptoms and past medical history. They may also be unwilling to cooperate with physical examination and tend not express pain or discomfort in the usual ways. It can therefore require much more time, patience and skill to assess such patients. One commonly used option is to take a collateral history from a family member or carer. However, it must be remembered that collateral histories are not always a source of accurate and impartial information. Clinicians should be careful not to break patient confidentiality unnecessarily when discussing patients with others, and specifically should be alert to the possibility of 'elder abuse'.

Delirium, particularly the hypoactive variant, represents a specific challenge that is often currently missed or overlooked especially in the busy ED environment (6). Recognition of 'brain failure' and prioritising investigation and management should receive the same clinical urgency as respiratory or renal failure. The presence of delirium is not a diagnosis in itself, but in the vast majority of cases, an indicator of an underlying health condition, including sepsis, electrolyte disturbance, drug effects, constipation and others. These health states require their own treatment along with other supportive measures for the delirious patient. Recognition of the change relies on collateral information about the individual and their usual cognitive state. Communication both to ED and subsequently ward staff that the patient has delirium, as well as explanation to their family and carers are critical in ensuring the patient is managed appropriately, recognising their vulnerable health state.

Older patients with dementia or delirium are also more likely to become agitated and sometimes aggressive. This is increasingly likely if they are cared for in an unfamiliar environment, if they have pain, an infection, electrolyte imbalance or dehydration, all of which are common in the fast-paced and busy emergency setting. Doctors may consider using pharmacological sedation, however this should be avoided and only used as a last resort; not only do anti-psychotics mask the underlying conditions, but the carry significant side-effects in older people, not least Parkinsonism and stroke risk. Each situation needs to be assessed on an individual basis with investigation into the likely cause of confusion, so that this can be appropriately treated. If further action is required, the least restrictive options should always be used first. Measures such as one-to-one nursing and improving the environment with familiar-looking possessions, photographs and people in the room should be attempted before the use of sedation or restraint. A pragmatic solution might be to ensure that people with delirium that require admission are moved rapidly to a definitive care setting, and do not 
spend a long period waiting in the ED, or ensuring that a bespoke area of the ED is made available for such patients.

\section{Best interests}

When making best interest decisions healthcare professionals must be mindful to ensure that they are genuinely considering the best interests of the patient and not confusing this with their personal view of the situation or the wishes of the patient's family. Time and care must be taken to consider the patient's past and present thoughts and feelings regarding the decision to be made, for example if they have made it clear to family members that they would not wish to be resuscitated should they die. Individuals may also have made Advance Care Plans or Advance Directives which must be considered, and may have appointed a Lasting Power of Attorney (LPA) who of course must be consulted. Any cultural or religious beliefs and values which the patient holds should also be taken into consideration, although care must be taken to ensure that assumptions regarding religion, culture, age or medical conditions are not made. Many patients, for example, may have the same diagnosis such as dementia, but this condition impacts differently on the quality of life of each individual and so it should not be assumed that the best interest decision is the same for every individual with dementia.

In order to make a best interest assessment, family members and people closest to the individual should be consulted as they may be able to provide useful information regarding the patient's wishes. However, the assessment should focus upon the best interests of the patient and not confuse this with the wishes of the family. This is not only important from the ethical perspective but also avoids placing undue pressure on families who may feel burdened by a perception that they are deciding the fate of their loved one. In practice, they are not making the decisions but are instead helping the clinical team best understand what their loved one would have wanted. This difference is subtle, but important to note and to explain to families during the decision making process as necessary.

\section{Advance care planning}

Advance care planning is one possible means by which people can exert greater control over their treatment, often, but not exclusively relating to end of life care issues. Advance Care Planning (ACP) is a process of discussion about future care between an individual and their care providers, irrespective of discipline(7).

The goals of advance care planning include:

- ensuring that clinical care is in keeping with patient preferences when the patient has become incapable of decision making (loss of capacity);

- improving the health care decision making process by facilitating shared decision making;

- improving patients' well-being by reducing the frequency of either under or over treatment.

ACP discussions may lead to an advance statement (a statement of wishes and preferences), an advance decision to refuse treatment (ADRT - a specific refusal of treatment(s) in a predefined potential future situation, also known as an 'Advance Directive')) or the appointment of a personal welfare Lasting Power of Attorney (LPA) or proxy. All or any of these can help inform care providers on the individual's best interests should the individual lose capacity. Equally there may be no specific output, though the discussion itself may hold intrinsic value. Some individuals will not want to engage in advance care planning discussions, and it is important not to pressure these individuals in to unwanted discussion. 


\section{Decision making - key issues}

The previous section outlined the ethical framework that needs to be considered when arriving at key decision in the emergency department settings (or indeed any context); the following factors will be especially relevant in the ED and should be taken into account when arriving at an ethical decision.

\section{Patient-related issues}

- Pain, fear, confusion, sensory impairment, expectations

- Older people may be concerned about the burden of their own illness on their family

- Carer strain - patients might be carers themselves and be more concerned about the person they care for than themselves

- The patient's gender, race, culture, sexual orientation, religion, beliefs and values

- Individuals should be encouraged to choose who they would wish to be included in the discussion

\section{Key informant issues}

It is particularly helpful if an older person attends the Emergency Department with a close friend, carer or relative who knows them well. This not only facilitates an exploration of the individuals usual physical and cognitive functioning, helping the assessing clinician to identify the impact of the acute presentation on the individual, but can also help in decision-making around suitability for discharge. They can provide an insight into the individual's capacity to cope with the acute insult and the level of care required. Without such information it is difficult not just to determine the severity of the current condition, but also to make informed decisions. The concern is that the natural tendency of the assessing clinician is to make the perceived 'safest' choice and opt to admit the individual as the uncertainty of their home situation makes hospital seem like the preferred option.

\section{Professional-related issues}

- The professional's own personal experience and beliefs. For example, if the professional has strong views on end of life care, influenced by their own religious beliefs, they should ensure that they do not impose their views on their patient. If there is a conflict of interest, a different professional opinion may be required.

- Training and experience - complex decision making should be led by an appropriately trained professional who has rapport with the individual and, where necessary, supported by a professional with relevant specialist knowledge

- The professional should have adequate knowledge about the disease, treatment and the particular individual to be able to give the patient all the information needed to express their preferences.

\section{System factors - patient flow versus individual patient care}

The traditional perspective is that a clinician's primary concern should be the patient before them, but increasingly clinicians are being expected to develop a broader perspective, including responsibility for all patients in the system, especially in the ED context.

Consider for example, clinicians as mangers - such as commissioners (those that commission services and control funding) being required to make difficult decision about resource allocation. A more direct example might be the clinician in the urgent care setting who needs to weigh-up the risk and benefits of admission for an individual patient, but also the broader patient population. There is a growing evidence base pointing towards the harms associated with long delays in emergency departments, and the consequences this has upon individual patients(8); it is not simply a 'managerial' issue. And whilst more beds might appear to be a solution, inevitably this needs to be traded off against the resource requirements, but also the 
process of care in the beds. Simply waiting in a hospital bed does not do any good and may well do harm (e.g. from deconditioning(1)), so it is improving the efficiency of care processes that is $\operatorname{key}(9)$.

\section{Ethics in practice in the Emergency Department}

\section{Design principles}

The Emergency Department is a highly challenging environment not entirely conducive to the assessment and management of frail older people. Physical factors include noise, bright lights, frequent movement and often other patients with behavioural disturbance, either from confusional states or toxicity from disease, drugs or alcohol.

Space is generally limited, and design is centred around improving patient flow, rather than orientation for individual patients. Identifying the bathrooms and accessing them can be challenging, particularly for those with restricted mobility or requiring aids.

To counter these issues, Geriatric Emergency Departments have evolved, mainly in North America(10). There are parallels here with paediatric EDs - separate services for population with particular needs. Whilst this idea might appear attractive, it does present some ethical challenges: what interventions are available? What is the cost of running a parallel service and could this be deployed more effectively? What about those people who are unable to access a separate ED?

In the UK, The Silver Book was created to draw together the interdisciplinary evidence-base to support the delivery of more effective acute and emergency care for frail older people(11). It identified eight core standards of care and then makes recommendations specific to individual assessment settings, four of which are directly applicable to the Emergency Department(11):

1. There should be a distinct area in Emergency Departments which is visually and audibly distinct that can facilitate multidisciplinary assessments

2. All units should have ready access to time critical medication used commonly by older people, such as Levo-Dopa

3. If a procedure is required for a person who is confused, two health care professionals should perform the procedure, one to monitor, comfort and distract, and the other to undertake the procedure; carers and/or family members should be involved if possible; cutaneous anaesthetic gel should be considered prior to cannulation, particularly if the person is confused.

4. All urgent and emergency care units should have accessible sources of information about local social services, falls services, healthy eating, staying warm, benefits and for carers of frail older people

So the main ethos of this guidance was to make the entire ED 'frail-friendly' rather than constructing distinct Geriatric EDs. In the authors' view, embedding frailty principles into EDs is a more logical long-term solution given the ageing demographic. The following sections illustrate some practical examples of how a frail friendly ED can be constructed.

\section{Service configuration}

\section{Patient streaming}

The first challenge is to establish who should receive such specialist assessment and who should remain within mainstream Emergency Department assessment procedures. There are a range of approaches which can be adopted to aid decision-making, each of which carries ethical issues. 
1. Risk scores and frailty scales to identify those at the highest risk of adverse outcomes

A number of risk scores have been tested in the ED with the aim of identifying those older people at the greatest risk of adverse outcomes, and then focusing services at this sub-group. Unfortunately the existing tools have limitations when operationalised in the ED, namely that they are not always simple to use, and their 'diagnostic accuracy' is poor (Area Under the Curve $<0.7)(12)$; examples include the Identification of Seniors At Risk (ISAR) and the Triage Risk Stratification Tool (TRST)(10-14).

The consequences of a screening process using such tools, is that some older people who might benefit from a CGA type intervention will be missed, whereas others who might not require it will be 'over-treated'. Although there are limited direct harms from over-exposure to CGA, there remains an issue about justice with limited resources potentially being directed away from the most needy.

\section{Age-related criteria}

Age is the simplest screening criteria, but there are many older patients who are not frail - and many younger patients who are frail - so it is also imperfect as a means of allocating to CGA or frailty services.

One potential danger from an age-guided streaming criterion is the potential to deny patients access to specialist care. In particular, time-critical interventions such as thrombolysis for acute stroke or primary percutaneous coronary intervention (PCI) for ST-segment myocardial infarction are both life-saving evidence-based interventions. To deny patient access or contribute to a delay in assessment would represent a form of age-related discrimination which cannot be justified. Furthermore, while many older adults will benefit from a multidisciplinary co-ordinated approach, some will have single-organ pathology requiring specialist management. Clearly at the level of the Emergency Department the crucial decision is where the patient needs to be for their ongoing management. Beyond this if geriatric services embrace the responsibility of managing complex older patients, they must also have effective relationships with specialists and a willingness to consult to ensure their patients benefit from the most contemporaneous, evidence-based management approaches.

3. An 'integrated approach'

Even if there were perfect criteria for assessing those patients most likely to benefit from geriatric services, it is unlikely that there will be sufficient resources for geriatric services alone to tackle the care of frail older people. So an alternative approach is to embed geriatric principles and practice into existing services - maintaining the focus on frailty, but generalising it in such a way that it becomes 'everyone's business'. Geriatric in-reach allows the specialist team to control their own workload and to select patients they think will benefit from their assessment. It requires an initial risk score to identify those most likely to benefit, supported by a more individualised assessment, and should generate effective communication between them and the Emergency Department team.

Frailty attuned clinical care

The ultimate goal of any selection or streaming of older people in the Emergency Department is to make appropriate decisions about their needs when presenting to secondary care services. Time pressures do not always allow for the needs of complex older people to be addressed in detail, with admission seen as the most obvious response to the complexity. However, an emergency department that embeds the principles of Comprehensive Geriatric Assessment (CGA) will be well placed to undertake an initial holistic assessment that can facilitate better, patient centred decision making. Comprehensive Geriatric Assessment is defined as: 
"a multidimensional, interdisciplinary diagnostic process to determine the medical, psychological and functional capabilities of a frail older person in order to develop a coordinated and integrated plan for treatment and long-term follow up"(13).

There is high quality evidence supporting the benefits of CGA as an effective intervention for older adults as a means of reducing rates of deterioration, death and institutionalisation compared to those receiving standard medical care(14-16). The evidence to-date points towards defined ward areas delivering CGA being more effective than liaison services (14-17), which is challenging to delivered in the ED setting. There are emerging models of appear to show improved service related outcomes (e.g. more people being safely managed at home)(18-20), but the optimal configuration is as yet unclear. Nevertheless it appears intuitive that service provision built upon the principles of CGA is likely to better meet the needs of frail older people accessing EDs.

Maintaining dignity in the ED

Dignity is an issue that does not just apply to older people, but to all patients in all healthcare settings, including the emergency department. However, there are several reasons which mean that older people in emergency departments are more vulnerable and that must have greater vigilance and act to protect their dignity.

Take the example of a patient who is seen in their cubicle by a doctor who exposes them for an examination but forgets to cover them up again once finished. Older patients who have cognitive or sensory impairments or who are acutely confused may not be fully aware that they have been left exposed following an examination. Similarly, older patients with communication difficulties, for example in dementia, delirium or following a stroke, may not be able to express that they have been left exposed, whilst a patient with severe arthritis or a broken limb following a fall may not be able to cover themselves.

\section{Ethical assessment}

When patients are assessed, care must be taken to ensure that a thorough assessment is made and that decisions are not made based upon assumptions about a patient or their preexisting illnesses. Although past medical history and current co-morbidities are significant factors in managing risk, for example suitability for anaesthesia, care must be taken to explore their impact further, especially if medical records are not available for corroboration, which is often the case in the ED. Caution must be exercised in the use of qualifying terms such as 'severe' or 'end-stage' using these only in reference to formal markers of disease severity. For example: heart failure classified using the New York Heart Association (NYHA) classification or chronic obstructive pulmonary disease classified with reference to FEV1, exercise tolerance, frequency and severity of exacerbations, past and previous treatment, rather than the admitting doctors impression of severity. This is magnified when decisions about the aggressiveness of treatment and escalation of care are being made. Such conversations should involve the patient, if able, accounting for their views and preferences. It should avoid individual doctors' judgements of the quality of life an individual has, particularly in the face of life-sustaining treatment. It is not for healthcare professionals to decide 'whose life is worth saving' or to guess at our perceptions of living with chronic disability. There is good evidence to support the view that individuals living with chronic disease wishes change over time(21) and the only reliable way to establish that is to engage in individual discussion.

Abuse

Whenever an older and potentially vulnerable patient is admitted to the emergency department, staff should be alert to the possibility of abuse. Abuse is defined as "a single or repeated act or lack of appropriate action, occurring within any relationship where there is an expectation of trust, which causes harm or distress to an older person". A 2007 UK study 
estimated a prevalence rate of abuse as 1 in 40 people over the age of 65 . Signs of abuse include patterns of injuries which are likely to be non-accidental, differing versions of events between patients and another individual and the vulnerable patient seeming to appear scared or withdrawn when certain individuals are present.

Clinicians working in these areas will come across cases of abuse and have a responsibility to take immediate and appropriate action, just as with child or domestic abuse.

\section{Palliative and end of life care}

In the oldest old admission to hospital is associated with a mortality rate of $46 \%$ at one year(2). A key challenge for staff in the Emergency Department is in the assessment and management of patients requiring supportive and palliative care. This ranges from the acute presentation of an individual with an un-survivable pathology, as well as those with established disease who are nearing the end of their life. Each scenario presents different challenges. In the former case, the rapid identification of the problem and explanation to the patient, where possible, and their family and carers are key to determining the priorities for ongoing care. In the latter, the challenge is establishing wishes and views at a time of crisis and often associated stress. The process of dying is highly variable between pathologies and not something with which many of the public have experience. It is unsurprising therefore that the Emergency Department receives patients close to the end of life. The challenge for staff there is to ensure the unaddressed need which has led to the attendance is assessed and support provided. It is also vital we acknowledge the unpredictability of medicine and do not deny such patients access to acute and emergency care services.

Advance Care Planning (see above) can play a useful role in helping manage these challenges, although uptake in clinical practice has been comparatively low $(22,23)$. A key barrier identified in a qualitative study of professionals in relation to ACP in dementia was concern that the current provision of healthcare services does not always allow us to meet the preferences individuals may express in an $\operatorname{ACP}(24)$. This challenge is real and relies on teams caring for patients to access information, support and services out with the hospital setting.

ED staff should be alert to the possibility of Advance Care Plans existing, and if possible develop systems to ensure that they are notified if a patient holds such a document. In some countries, doctors are obliged to consult a central register of ACP documents when making best interests decisions(25). Labelling of case notes regarding the presence/absence of ACP documents may only be accurate on $60-90 \%$ of occasions $(26,27)$. Where an advance care plan exists, this can help to guide professionals providing care to allow the wishes and preferences of the individual to be met, provided these are also their wishes at that time. The challenge can be when relying on surrogate decision-makers, such as family or carers, who frequently do not provide an accurate reflection of the views of the patient, with incorrect prediction in a third of cases in one study(28). Hospitalisation can be regarded as a sentinel event that heralds an intensive period of health and social care service use(29), so an ED attendance might be used as a prompt to consider Advance Care Planning as part of the ongoing transfer of care.

Staff in the Emergency Department must also be offered training in basic aspects of Palliative and Supportive Medicine as taking this approach often diverts them from their usual 'modus operandi' of preserving life as the ultimate priority. Establishing good links with the hospital palliative care team can also help to facilitate patient preferences for care when they present to hospital.

\section{Communicating effectively and sharing information}

A challenge common to different health settings is the effective sharing of information. This is particularly relevant in establishing a reliable past medical history, record of current and 
previous medications and allergies, as well as accessing prior investigations. Such information can supplement the details known by the patient and carer and facilitate better-informed decision-making and reduce avoidable error by making inappropriate recommendations of medications known to have adverse effects and reduce waste by repeating investigations. The benefits of accessing information are higher in relation to decisions such as those around resuscitation and the levels of treatment which patients accept in a life-threatening situation (e.g. non-invasive ventilation, intravenous antibiotics etc). Healthcare professionals are often uncertain about such decisions, particularly if original documentation is not available. In such situations, the presumption is rightly to intervene, however this can often be due to a lack of information and uncertainty. In the highly digitalised age moves towards co-ordinated electronic records spanning primary, secondary and community care services are essential. Similarly when an individual is transferred from the emergency department, effective communication with their other health and care providers is a useful way of acting on the recommendations made and ensuring care is contemporaneous.

Nursing care of the dying in ED

By acting as the front door, the ED is accessible to anyone when symptoms appear to be out of control including some who are actively dying. ED staff are exposed to death and dying on a regular basis(30). Death is never an easy concept to deal with or understand, especially so when it is sudden and unexpected. The available literature has reported concerns of those dying in the acute hospital but there is limited material on older people dying in the $\operatorname{ED}(30$, 31). Families experiencing grief rely on nursing staff for comfort and answers. Evidence suggests families will often recall the initial interactions months later(31). Offering support can be very challenging in a fast-paced environment. Relationships that can be built up over days and weeks in the inpatient or hospice setting must be formed in hours or minutes in the emergency department. Many nurses feel that EDs are not the most appropriate place for patients to die, given the focus on saving lives, and resuscitation over end of life care $(30,32)$. Often ED clinicians do not have the benefit of knowing their patient's values and wishes to guide their clinical decisions, therefore death may be perceived as preventable in the absence of full picture, which can lead to feelings of guilt or defeat.

Often, standard practice in cardiac arrest scenarios is to administer cardiopulmonary resuscitation in the absence of DNAR, regardless of the illness. Staff nursing the patient often go through a series of emotions, anxieties related to their own personal loses and fears of death. The ability to cope with one's emotions has an impact on how one interacts with patients and families. Studies have suggested there is variability in how nurses manage their emotions and how they manage the dying process $(33,34)$. Nurses should be supported and given adequate time and resources to debrief thoughts and feelings regarding death and dying. Most current ED designs do not have private facilities or quite area to let families grieve and offer emotional support. With the increasing volume of patients seeking attention in ED, this quiet area may be sacrificed to accommodate other patients, causing a dilemma to nurses offering care(33).

Effective debriefing and coping often are difficult in a fast-paced environment. At times, acknowledging emotion and admitting sensitivity is sufficient to allow an emergency nurse some sense of closure. Simple acknowledgment, however, should not replace the process of allowing an emergency nurse the adequate time and resources to debrief and deal with his or her thoughts and feelings regarding death and dying. 


\section{Summary, conclusion and future directions}

In this article, we have illustrated the ethical principles that should govern good clinical practice. We have used the Emergency Department as a specific setting where the challenges of delivering ethical care are especially pronounced. We have particularly focussed upon the role of capacity assessment and best interest decisions, as these can be particularly difficult in the Emergency Department setting, for example in patients with confusional states. We offer some practical guidance on how to address such scenarios.

We have also broadened the perspective from the individual patient to the urgent care axis as a part of a whole system. The aim of this is to encourage clinicians working in EDs to consider themselves as members of the broader healthcare system, and consider the implications of their clinical practice for others who will be caring for the same patient, often during the same episode of care, in other parts of the system. We have done this as there is much to be gained, especially for older people with frailty, through ethically sound, whole systems approaches.

We have promulgated the notion of 'frail friendly Emergency Departments' that are ethically designed and capable to deliver frailty attuned care. This is because if European healthcare systems are to respond to the increasing numbers of older people with frailty, there will need to be a step-change in the provision of urgent care. Whilst we fully accept that urgent care is not synonymous with whole system care, the Emergency Department is a critical component of the patient pathway. This philosophy aligns with European efforts to create a 'social movement' around Geriatric Emergency Medicine, exemplified by the productive collaboration between the European Society of Emergency Medicine, and European Society of Geriatric Emergency Medicine (http://www.eugms.org/research-cooperation/special-interestgroups/geriatric-emergency-medicine.html). 


\section{References}

1. English KL, Paddon-Jones D. Protecting muscle mass and function in older adults during bed rest. Current Opinion in Clinical Nutrition and Metabolic Care. 2010;13(1):34-9.

2. Clark D, Armstrong M, Allan A, Graham F, Carnon A, Isles C. Imminence of death among hospital inpatients: Prevalent cohort study. Palliative Medicine. 2014;28(6):474-9. 3. Beauchamp T CJ. Principles of Biomedical Ethics. New York: Oxford University Press; 1979. p. 56.

4. Department for Constitutional Affairs. Mental Capacity Act London2005 [cited 2005 16th May 2005]. Available from: http://www.hmso.gov.uk/acts/acts2005/20050009.htm.

5. Boyle G. The Mental Capacity Act 2005: promoting the citizenship of people with dementia? Health Soc Care Community. 2008;16(5):529-37.

6. Lamantia MA, Messina FC, Hobgood CD, Miller DK. Screening for Delirium in the Emergency Department: A Systematic Review. Ann Emerg Med. 2013.

7. NHS End of Life Care Programme. Advance Care Planning: A Guide for Health and Social Care Staff. London: 2007.

8. Carter EJ, Pouch SM, Larson EL. The Relationship Between Emergency Department Crowding and Patient Outcomes: A Systematic Review. J Nurs Scholarsh. 2013;46(2):106-15. 9. Inouye SK, Bogardus ST, Baker DI, Leo-Summers L, Cooney LM. The Hospital Elder Life Program: A model of care to prevent cognitive and functional decline in older hospitalized patients. Journal of the American Geriatrics Society. 2000;48(12):1697-706.

10. Geriatric Emergency Department Guidelines. Annals of Emergency Medicine. 2014;63(5):e7-e25.

11. Banerjee J, Conroy S. Quality Care for Older People with Urgent \& Emergency Care Needs2012. Available from:

http://www.bgs.org.uk/campaigns/silverb/silver book complete.pdf.

12. Graf CE, Giannelli SV, Herrmann FR, Sarasin FP, Michel J-P, Zekry D, et al. Can We Improve the Detection of Old Patients at Higher Risk for Readmission After An Emergency Department Visit? Journal of the American Geriatrics Society. 2012;60(7):1372-3.

13. Rubenstein LZ, Rubenstein LV. Multidimensional assessment of elderly patients. Adv Intern Med. 1991a;36:81-108.

14. Ellis G, Whitehead M, Robinson D, O'Neill D, Langhorne P. Comprehensive geriatric assessment for older adults admitted to hospital: meta-analysis of randomised controlled trials. British Medical Journal. 2011;343(d6553).

15. Baztan JJ, Suarez-Garcia FM, Lopez-Arrieta J, Rodriguez-Manas L, Rodriguez-Artalejo F. Effectiveness of acute geriatric units on functional decline, living at home, and case fatality among older patients admitted to hospital for acute medical disorders: meta-analysis. BMJ. 2009;338(jan22_2):b50-.

16. Fox MT, Persaud M, Maimets I, O'Brien K, Brooks D, Tregunno D, et al. Effectiveness of Acute Geriatric Unit Care Using Acute Care for Elders Components: A Systematic Review and Meta-Analysis. Journal of the American Geriatrics Society. 2012;60(12):2237-45.

17. Deschodt M, Flamaing J, Haentjens P, Boonen S, Milisen K. Impact of geriatric consultation teams on outcomes in acute hospitals: a systematic review and meta-analysis. BMC Medicine. 2013;11:48.

18. Wright PN, Tan G, Iliffe S, Lee D. The impact of a new emergency admission avoidance system for older people on length of stay and same-day discharges. Age and Ageing. 2013.

19. Conroy SP, Ansari K, Williams M, Laithwaite E, Teasdale B, Dawson J, et al. A controlled evaluation of comprehensive geriatric assessment in the emergency department: the 'Emergency Frailty Unit'. Age and Ageing. 2013.

20. Pareja-Sierra T, Hornillos-Calvo M, Solís JR, Sepúlveda-Moya DL, Bassy-Iza N, Martínez-Peromingo FJ, et al. Implementation of an Emergency Department Observation Unit for Elderly Adults in a University-Affiliated Hospital in Spain: A 6-Year Analysis of Data. Journal of the American Geriatrics Society. 2013;61(9):1621-2.

21. Ditto P, Jacobson J, Smucker W, Danks J, Fagerlin A. Context Changes Choices: A Prospective Study of the Effects of Hospitalization on Life-Sustaining Treatment Preferences. Medical Decision Making. 2006;26:313-22.

22. Hughes $\mathrm{P}$, Bath $\mathrm{P}$, Ahmed N, Noble B. What progress has been made towards implementing national guidance on end of life care? A national survey of UK general practices. Palliative Medicine. 2010;24:68-78. 
23. Musa I, Seymour J, Conroy S. A Survey Of Older Peoples' Attitudes Towards Advance Care Planning. International Symposium on Supportive Care in Cancer; Miami2014.

24. Robinson L, Dickinson C, Bamford C, Clark A, Hughes J, Exley C. A qualitative study: Professionals' experiences of advance care planning in dementia and palliative care, 'a good idea in theory but.....'. Palliative Medicine. 2013;27(5):401-8.

25. van Asselt D. Advance directives: Prerequisites and usefulness. [References]: Zeitschrift fur Gerontologie und Geriatrie. Vol 39(5) Oct 2006, 371-375. Springer.; 2006.

26. Wallace J, Desbiens NA. Evaluation of a labeling system to indicate the presence of an advance directive in a hospital medical record. International Journal for Quality in Health Care. 2004;16(4):333-5.

27. Cohen-Mansfield J, Libin A, Lipson S. Differences in presenting advance directives in the chart, in the minimum data set, and through the staff's perceptions. Gerontologist.

2003;43(3):302-8.

28. Shalowitz D, Garrett-Mayer E, Wendler D. The Accuracy of Surrogate Decision Makers. Archives of Internal Medicine. 2006;166:493-7.

29. Woodard J, Gladman J, Conroy S. Frail older people at the interface. Age Ageing. 2010;39(S1):i36.

30. Bailey CJ, Murphy R, Porock D. Dying cases in emergency places: caring for the dying in emergency departments. Soc Sci Med. 2011;73(9):1371-7.

31. Norton CK, Hobson G, Kulm E. Palliative and end-of-life care in the emergency department: guidelines for nurses. J Emerg Nurs. 2011;37(3):240-5.

32. Bailey C, Murphy R, Porock D. Trajectories of end-of-life care in the emergency department. Ann Emerg Med. 2011;57(4):362-9.

33. Wodchis WP, Dixon A, Anderson GM, Goodwin N. Integrating care for older people with complex needs: key insights and lessons from a seven-country cross-case analysis. Int J Integr Care. 2015; 15:e021.

34. Tardy B, Venet C, Zeni F, Berthet O, Viallon A, Lemaire F, et al. Death of terminally ill patients on a stretcher in the emergency department: a French speciality? Intensive Care Med. 2002;28(11):1625-8. 